ASEAN Journal of Science and
Engineering Education

\title{
Study of LED Grow Light Driver Circuit for Indoor Strawberry Cultures: A Class Experiment
}

\author{
S. Sungwiset, T. Janprung, T. Pechsiri, S. Puengsungewan* \\ Department of Electrical Technology Education, Faculty of Industrial Education and Technology \\ King Mongkut's University of Technology Thonburi, Thailand \\ * Correspondence: E-mail: supachai.pue@kmutt.ac.th
}

\section{ABSTRACTS}

This journal presents the study of the led driver circuits for strawberry LED grow light. There are three driver circuits: A linear resistor circuit, a simple transistor circuit, and a boost converter circuit. Boost converter circuits are more efficient than direct drives and transistor drives. Because the circuit can be controlled from a $40 \mathrm{kHz}$ PMW signal that gives a more constant value and circuit stability. There is also less load in the circuit, making less loss in the circuit with a highefficiency value. When the voltage used is 12 volts, we got a value of Photosynthetic Photon Flux Density (PPFD) of 230 $\mu \mathrm{mol} / \mathrm{m} 2 / \mathrm{s}$. This circuit has very high efficiency (98\%). The boost converter is suitable for driving LED bulbs for strawberry planting.

\author{
ARTICLE INFO \\ Article History: \\ Submitted/Received 29 Jan 2021 \\ First revised 22 Feb 2021 \\ Accepted 24 Feb 2021 \\ First available online 26 Feb 2021 \\ Publication date 01 Mar 2021 \\ Keyword: \\ Boost converter, \\ LED driver circuit, \\ LED grow light, \\ Strawberry culture.
}




\section{INTRODUCTION}

Strawberry is one kind of major economic crop in the world. Currently, the cultivation of strawberries is widely cultivated commercially. There are two types of strawberry cultivation. It is mainly manual labor and strawberry cultivation in the form of smart farms. Artificial Intelligence and Robot Technology It is widely applied to modern agriculture. including strawberry farms (Ge et al., 2017; Yu et al, 2020). Hydroponics is also an option for farmers who want to grow strawberries without soil. Hydroponics can be grown both indoors and outdoors (Suroto, 2021).

Light-emitting diode (LED) is a technology that is used in a wide variety of applications. For agriculture, LED lighting is used as a light source, effectively replacing sunlight. Currently, there are studies on how to use LED lighting in greenhouses to increase productivity. The key point that makes LED light so popular is that LED light consumes less power than other forms of artificial light sources. In addition, LED technology has a longer lifespan lowering the cost of cultivation. According to research on the energy-saving of LED light when used with plants in greenhouses, it has been found that up to $30 \%$ of electricity can be saved (Jiang et al., 2020).

Although the highlight of LED technology is energy-saving and the long life of the lamp. However, the lamp driving circuit must be considered. Theoretically, the LED is structured from the PN junction, so driving the LED to be bright requires a DC voltage. Therefore, the LED driver circuit is important for energy saving and lamp life as well. over the past several decades. Researchers from around the world have presented their research on LED driver circuits to achieve high efficiency using power electronics technology. The strength of power electronics technology is that it can drive various types of loads. efficiently and compactly (Olabo et al., 2021). An LED driver using a Trian-type switch was introduced in 2015. This circuit structure has a simple circuit structure. Moreover, its efficiency is higher than commercially available linear circuits (Moon et al., 2015).

For today's most efficient LED driver technology, researchers around the world are interested in Boost Converter drive circuits. Another important feature is that the drive unit generates less heat. Due to the low loss, the power dissipation is very low, theoretically, the Boost converter drive circuit is more than 90\% efficient (Kimball et al., 2004; Sien et al., 2012; Abdelmessih et al., 2020).

Application of LED Lighting technology in strawberry cultivation There are issues to keep in mind. both the quantity and the spectrum of light Moreover, the radiated heat may affect the growth of strawberries, which are low-temperature plants. This paper presents a comparative study of the light and thermal issues emitted by the drive unit. The study divided circuits into 3 types: Linear resistor, simple transistor circuit, and a boost converter circuit.

\section{METHODS}

To study the LED driver circuit for strawberry cultures, there are three driver circuits: A linear resistor circuit, a simple transistor circuit, and a boost converter circuit. Then to calculate Photosynthetic Photon Flux Density (PPFD) values suitable for plants, we calculated the number of tubes used in each circuit to be designed in the circuit. Finally, we took each circuit to compare efficiency. 


\section{RESULTS}

\subsection{Presentation of The Three Driver Circuits}

There are three driver circuits: a linear resistor circuit, a simple transistor circuit, and a boost converter circuit. shown in Figures 1, 2, and $\mathbf{3}$ respectively.

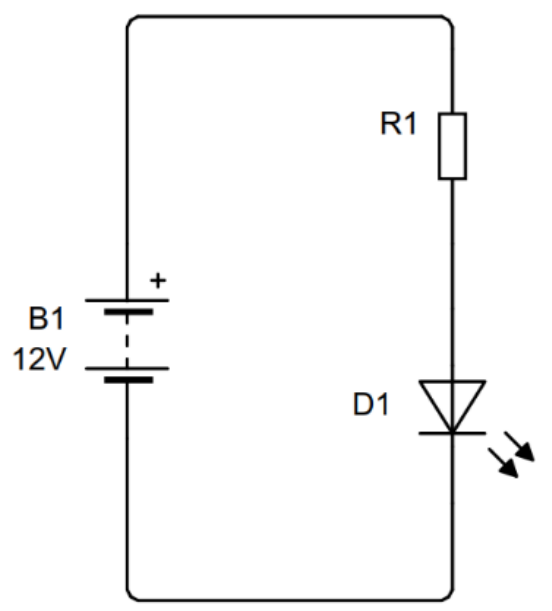

Figure 1. Linear resistor driver circuit.

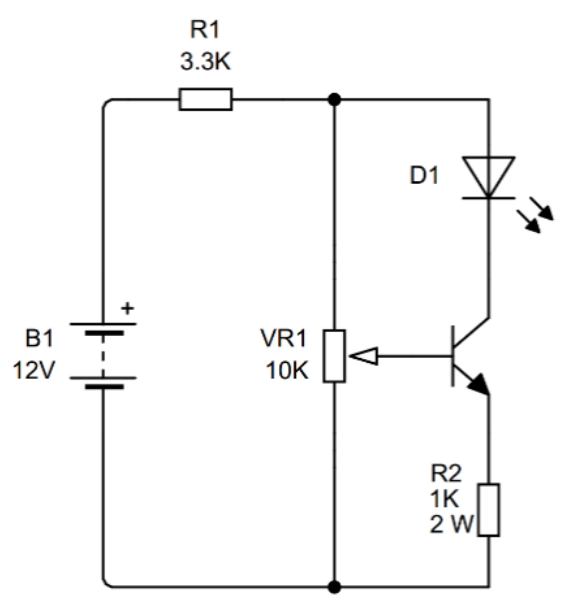

Figure 2. Transistor driver circuit.

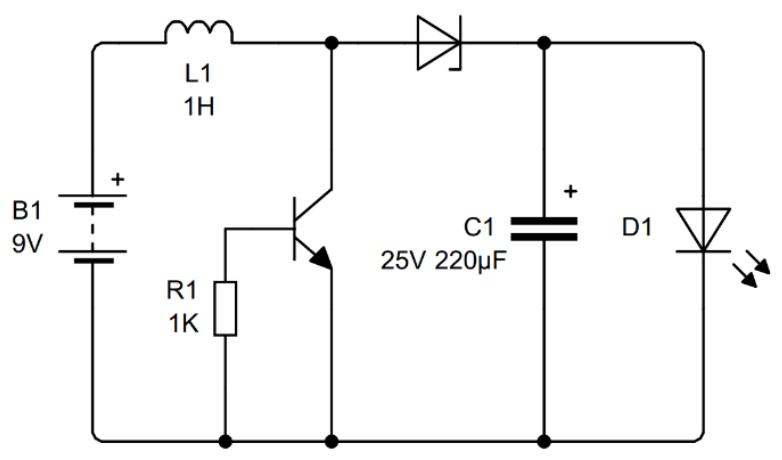

Figure 3. Boost converter driver circuit. 


\subsection{Linear Driver Circuit}

The principle of current flow is by using resistors to drop currents of red and blue. to have the same brightness by using a voltage switch of 12 volts. As a power supply to the circuit. Supply current through a $5 \Omega$ 30-watt resistor flows through the red LED and an $18 \Omega$ 10watt resistor flows through the blue LED shown in Figure 4.

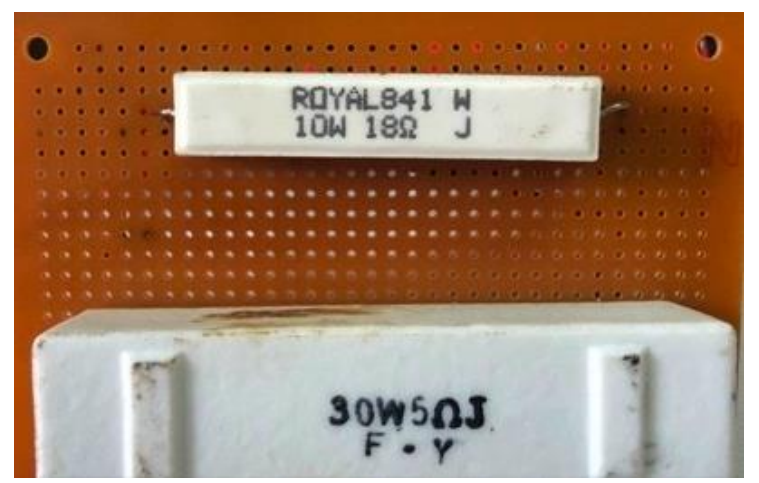

Figure 4. a $5 \Omega$ 30-watt resistor and an $18 \Omega 10$-watt resistor.

When getting luminance values from LED data in Table 1, calculate PPFD values so that the luminance values are suitable for strawberry trees. LUX values are 16 lux/luminance for the red bulb and 18 lux/luminescent for the blue bulb.

Table 1. 5mm Round LEDs Specifications.

\begin{tabular}{lccccccc}
\hline $\mathbf{5} \mathbf{~ m m}$ Round LEDs & Forward Voltage & \multicolumn{2}{c}{$\begin{array}{c}\text { Dominant } \\
\text { Wavelength }\end{array}$} & Luminous Intensity & $\begin{array}{c}\text { Viewing } \\
\text { Angle }\end{array}$ \\
\hline \multicolumn{1}{c}{ Emitting Color } & $(\mathrm{V}) \mathrm{IF}=20 \mathrm{~mA}$ & \multicolumn{2}{c}{$\mathrm{IF}=20 \mathrm{~mA}$} & \multicolumn{2}{c}{ (mcd) IF = 20 mA } & \\
& TYP & $\mathrm{MAX}$ & $\mathrm{MIN}$ & $\mathrm{MAX}$ & $\mathrm{TYP}$ & $\mathrm{MAX}$ & (degree) \\
Red & 1.8 & 2.3 & 620 & 640 & 4000 & 5000 & 25 \\
Blue & 3.2 & 3.4 & 465 & 475 & 5000 & 7000 & 25 \\
\hline
\end{tabular}

We used 5 red LEDs for getting 80 lux and used 1 red LED for 20 lux. So, when we want to get values equal to 100 lux, approximately 25 sets are made. After that, design the circuit into the PCB shown in Figure 5.

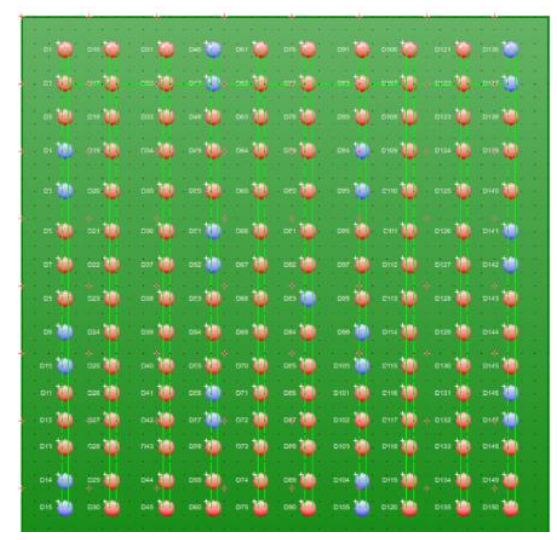

Figure 5. Designing LED tube for linear resistor Driver Circuit into the PCB and operate the electrical circuit. Get Lux $=2500$ PPFD $=221 \mu \mathrm{mol} / \mathrm{m} 2 / \mathrm{s}$. 
This circuit has a very high temperature at electric devices shown in Figure 6 . The circuit used 160 LED lamps, causing a lot of resistance in the lamp and the resistors in the circuit are $5 \Omega$ for 30 Watt and $18 \Omega$ for 10 Watt.

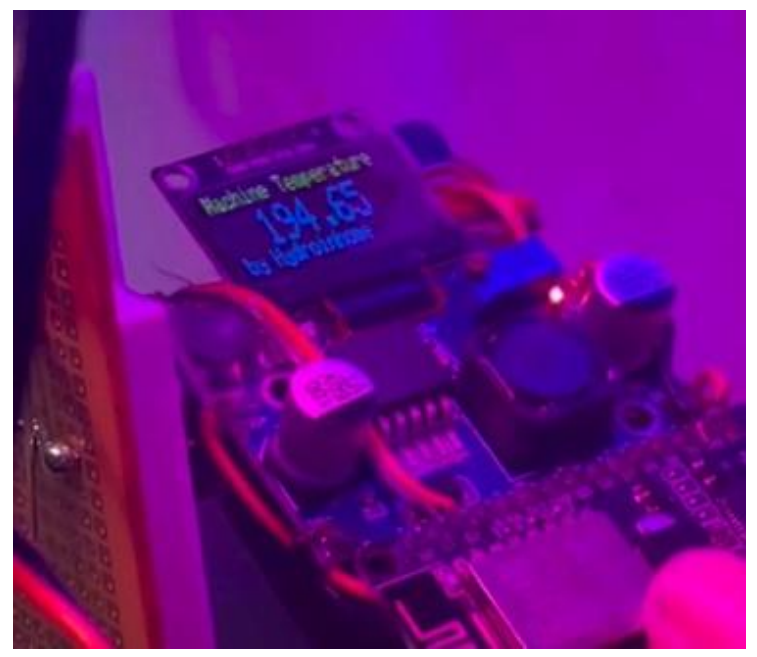

Figure 6. The temperature value from device electric in the linear resistor driver circuit.

\subsection{Transistor Driver Circuit}

The principle of the transistor circuit is to control the LED current by adjusting the collector current of the transistor. In a driving circuit experiment using a transistor as a current dimmer and then using a BC440 transistor. Transistor Bc440 can withstand more current than conventional transistors and can get dimmer with the current $V R$ of $10 \mathrm{k}$ to obtain the proper current IC total $=0.11 \mathrm{~A}$ and IE $=0.69 \mathrm{~mA}$. But, if using R1 that is more than $3.3 \mathrm{k}$ ?, it will cause the current to drop at R1. Thus, VR 10k? did not work and cannot flow dimmer. One transistor can drive 192 LED bulbs. After that, we designed the circuit into the PCB shown in Figure 7.

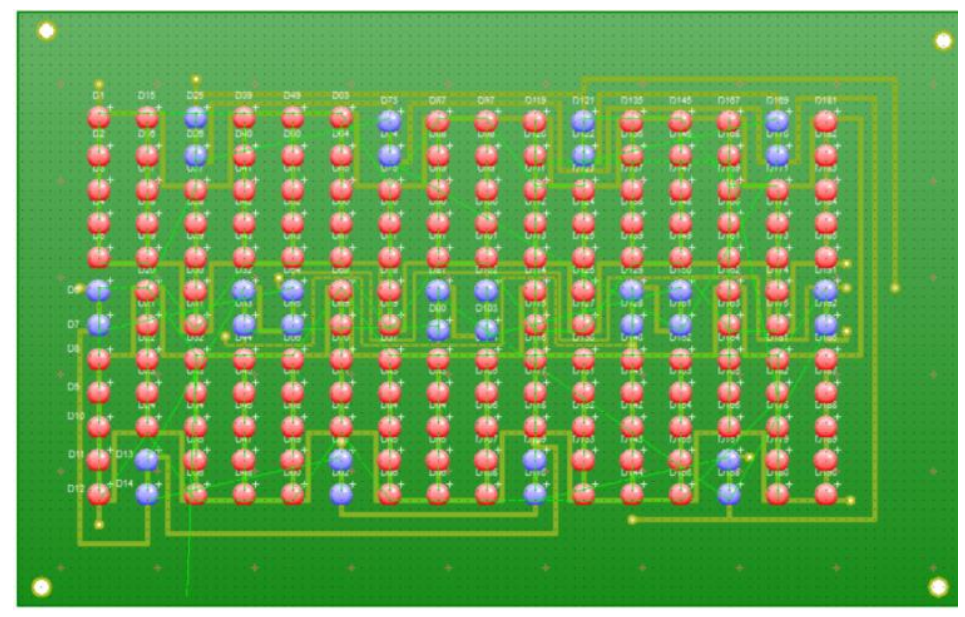

Figure 7. Designing LED tube for transistor Driver Circuit into the PCB and operate electrical circuit.

We measured the temperature of the plant's response to LED light, shown in Figure 8. We got a PPFD value of $217 \mu \mathrm{mol} / \mathrm{m} 2 / \mathrm{s}$. 


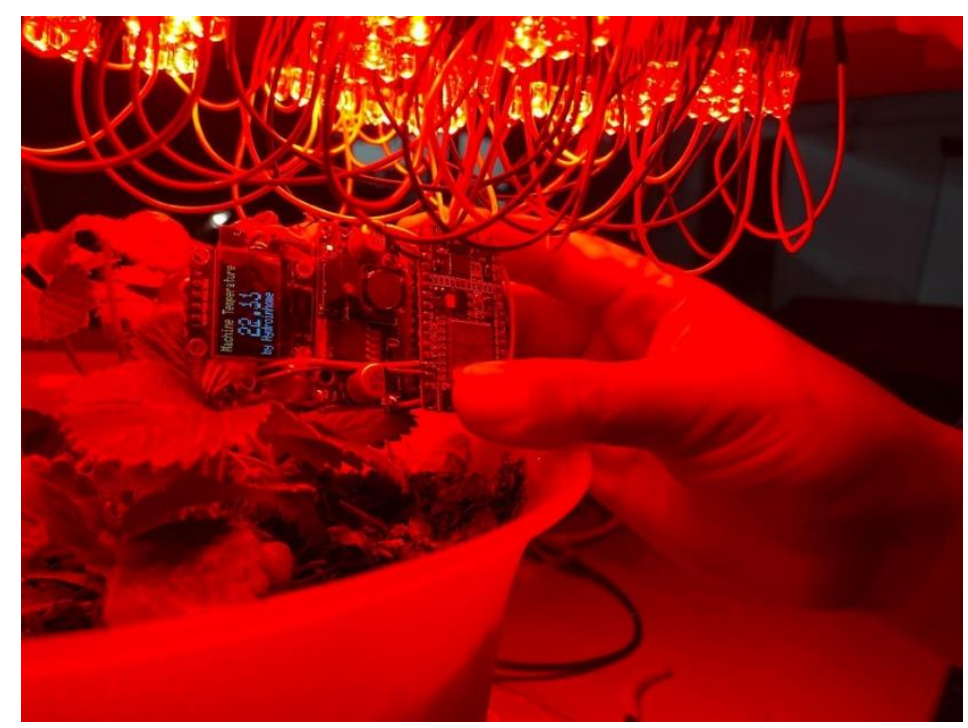

Figure 8. The plant's response to LED light.

\subsection{Boost Converter Driver Circuit}

The principle of the boost converter is controlled by PMW. It is used to dimmer the current to stabilize. When getting the Fs value suitable for doing, it will be Fs $=40 \mathrm{kHz}$, shown in Figure 9.

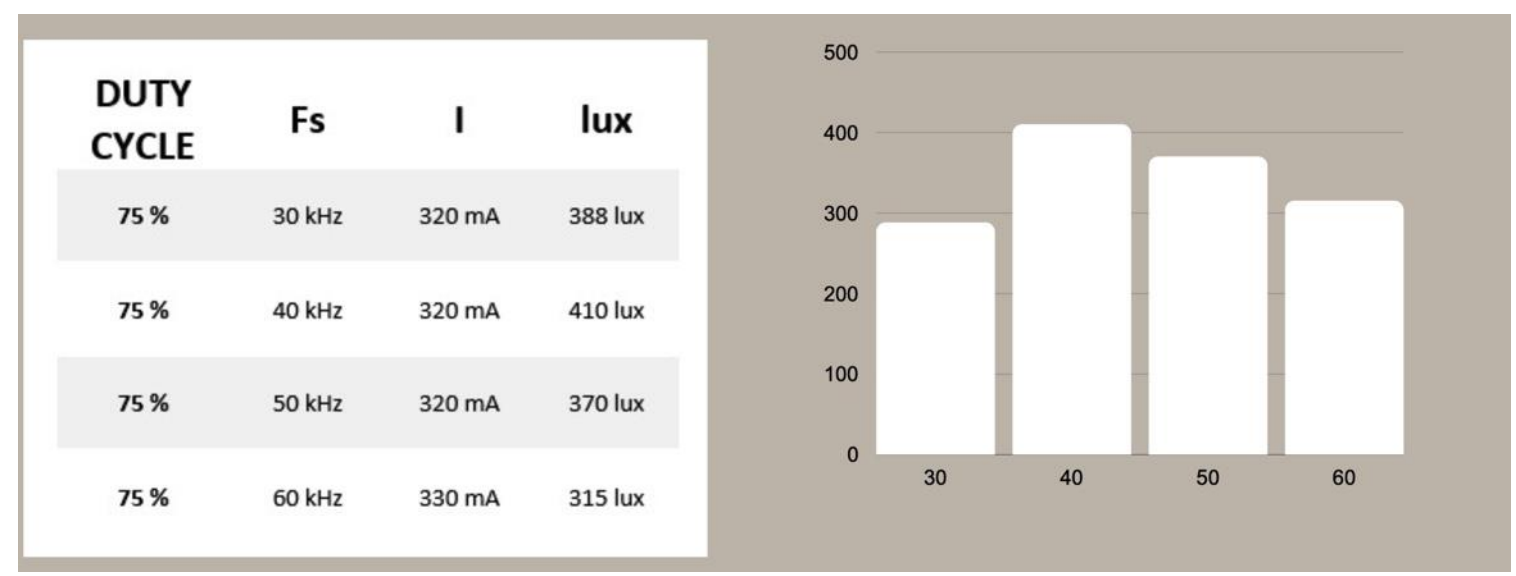

Figure 9. Comparison of frequency modulation from PWM signal affecting lighting.

When using a process for 5 LED bulbs with Vin $=1.5$ volts and Vout $=9.5$ volts, we made an experimental design to use the supply voltage $V=12$ Volts and Vout= $15 \mathrm{~V}$ for 8 LED red with 500 lux. The boost converter used only 58 LED tubes in the circuits. After that, we designed the circuit into the PCB, shown in Figure 10, and operate the electrical circuit. We obtained a PPFD value of $230 \mu \mathrm{mol} / \mathrm{m} 2 / \mathrm{s}$. The problem is a circuit issue due to the capacitor's value spanking or sudden charge-discharge phenomena. But it is solved using an apparatus with 25 volts and $220 \mu \mathrm{F}$. 


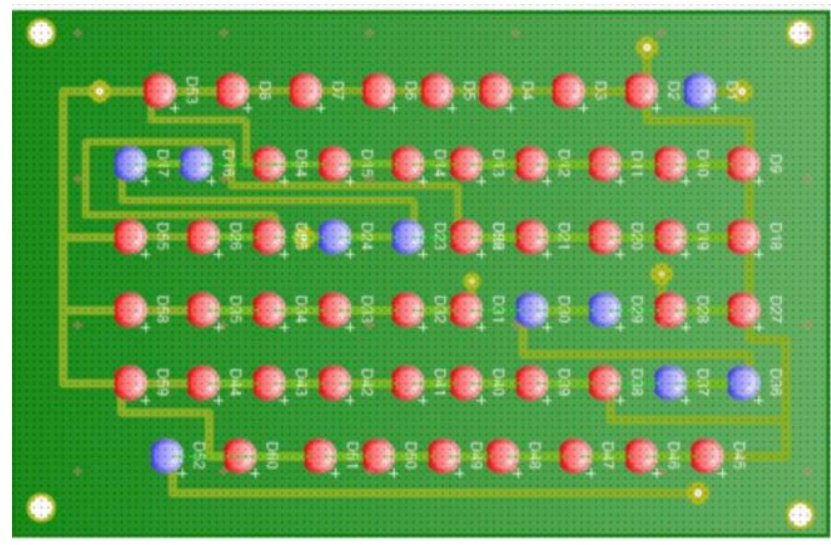

Figure 10. Designing LED tube for boost converter driver circuit into the PCB.

\subsection{Performance Comparison of 3 Circuits}

In Figure $\mathbf{1 1}, \mathbf{1 2}, \mathbf{1 3}$, and 14 a linear driver circuit efficiency is $22 \%$ and loss is $78 \%$, which is the circuit with the lowest efficiency. Transistor driver circuit efficiency is $44 \%$ and loss is $56 \%$, which is a circuit with moderate efficiency. Boost converter driver circuit efficiency is equal to $98 \%$ and loss is equal to $2 \%$, which is the most efficient circuit. So, it can be concluded that the Boots converter driver circuit has the best efficiency and is suitable for use.

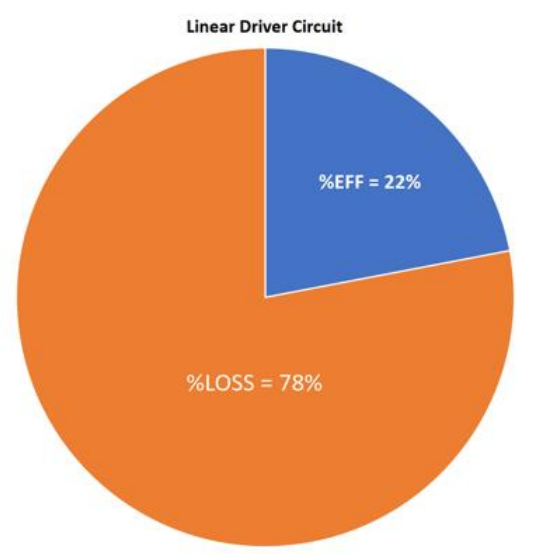

Figure 11. Performance comparison of 3 circuits based on the linear driver circuit.

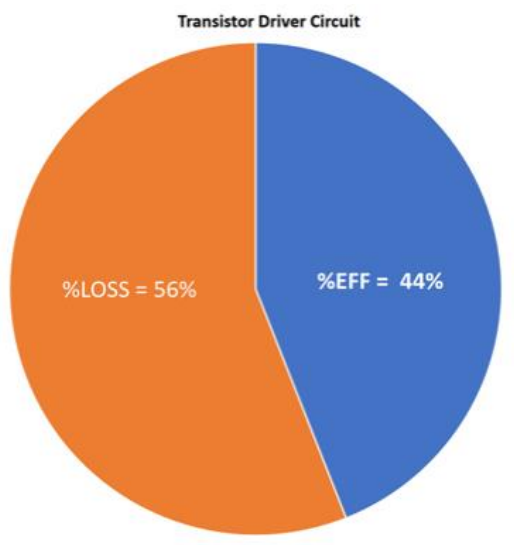

Figure 12. Performance comparison of 3 circuits based on the transistor driver circuit. 


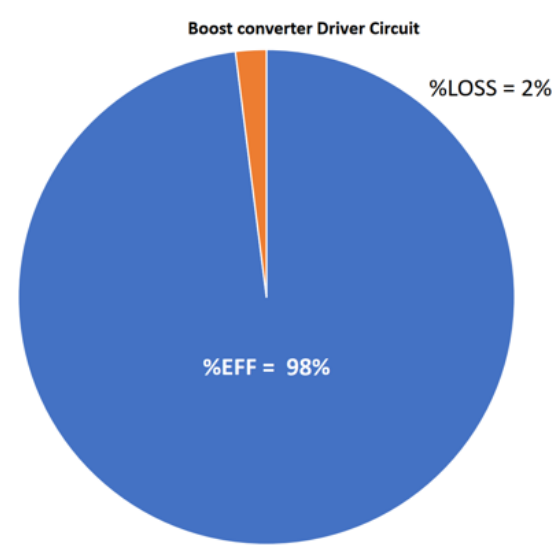

Figure 13. Performance comparison of 3 circuits based on the boo's converter driver circuit.

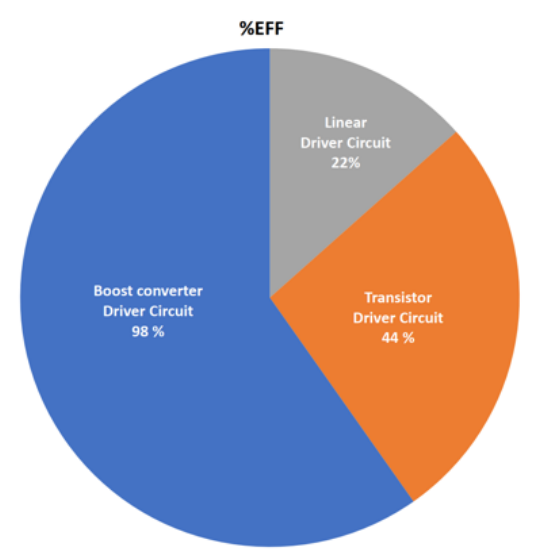

Figure 14. Performance comparison of 3 circuits based on efficiency.

\section{DISCUSSION}

Statistics for using Linear resistor driver circuits for 150 tubes, 125 red LEDs, 50 blue LED, Voltage of 12 volts, we got a value of PPFD $=150 \mu \mathrm{mol} / \mathrm{m}^{2} / \mathrm{s}$. The device in the circuit heats up to about $200^{\circ} \mathrm{C}$. This circuit has very low efficiency due to the high electrical load.

Statistics for using Transistor driver circuit for 192 tubes,160 red LEDs, 32 blue LEDs, Voltage of 12 volts, we got a value of PPFD $=217 \mu \mathrm{mol} / \mathrm{m}^{2} / \mathrm{s}$. This circuit has an efficiency of $44 \%$. The LED bulb driving circuit with a transistor is much more efficient than direct bulb driving as it reduces the resistor load or the resistor used in the circuit. Cycle efficiency is only $44 \%$, but the temperature of the equipment in the cycle is without heat consideration, and there is constant heat.

Statistics for using Boost converter for 58 tubes, 48 red LEDs, 10 blue LEDs, Voltage of 12 volts, we got a value of PPFD $=230 \mu \mathrm{mol} / \mathrm{m}^{2} / \mathrm{s}$. This circuit has a very high efficiency of $98 \%$. LED driver circuit with Boost converter circuit is more efficient than driving from Transistor driver circuit because it can control the circuit from $40 \mathrm{kHz}$ PMW signal that gives more constant value. Also, it brings stability to the circuit. The results of the 3 experiments from our circuits are shown in Table 2. 
Table 2. Results from three circuits.

\begin{tabular}{lccccccccc}
\hline \multicolumn{1}{c}{ Circuit } & Volt & \multicolumn{2}{c}{ Number of tubes } & PPFD & $\begin{array}{c}\text { Temperature } \\
\text { (degree } \\
\text { Celsius) }\end{array}$ & $\begin{array}{c}\text { PMW } \\
(\mathbf{k H z})\end{array}$ & \%EFF & \%LOSS \\
\hline $\begin{array}{l}\text { linear } \\
\text { driver } \\
\text { circuits }\end{array}$ & $12 \mathrm{~V}$ & 125 & 50 & 150 & 152 & 200 & - & 22 & 78 \\
$\begin{array}{l}\text { Transistor } \\
\text { driver } \\
\text { circuit }\end{array}$ & $12 \mathrm{~V}$ & 160 & 32 & 192 & 217 & 27.67 & - & 44 & 56 \\
$\begin{array}{l}\text { Boost } \\
\text { converter }\end{array}$ & $12 \mathrm{~V}$ & 48 & 10 & 58 & 230 & 25 & 40 & 98 & 2 \\
\hline
\end{tabular}

\section{CONCLUSION}

Boost converter circuits are more efficient than linear and transistor circuits. Control circuit using $40 \mathrm{kHz}$ PMW signal brings more constant condition. It is also less loaded. It makes less loss in the circuit with a high-efficiency value using a converter. This circuit has a very high efficiency (reaching 98\%). The boost converter is suitable for driving LED bulbs for strawberry cultures.

\section{ACKNOWLEDGMENT}

We would like to thank The Department of Electrical Technology Education, Faculty of Industrial Education and Technology, the King Mongkut's University of Technology Thonburi for allowing us to attend the conference ASEAN Symposium for Science, Engineering, Economics, and Education (ASSEEE), and we would like to thank the cooperation and active participation of the faculty and friends.

\section{AUTHORS' NOTE}

The authors declare that there is no conflict of interest regarding the publication of this article. Authors confirmed that the paper was free of plagiarism.

\section{REFERENCES}

Abdelmessih, G., Alonso, J., A. Dalla, M., Chen, Y., and Tsai, W. (2020). Fully Integrated Buck and Boost Converter as a High Efficiency, High-Power-Density Off-Line LED Driver. IEEE Transactions on Power Electronics, 35(11), 12238-12249.

GE, Y., Xiong, Y., Lins, G., and Johan, P. (2017). Fruit Localization and Environment Perception for Strawberry Harvesting Robots. IEEE Access, 7(2019), 147642-147651.

Jiang, J., Mohagheghi, A., and Moallem, M. (2020). Energy-Efficient Supplemental LED Lighting Control for a Proof-of-Concept Greenhouse System. IEEE Transactions on industrial Electronics, 67(4), 3033-3041.

Kimball, W. J., Flowers, L. T., and Chapman, L. P. (2004). Low-Input-Voltage, Low-Power Boost Converter Design Issues. IEEE Power Electronics letters, 2(3), 96-99. 
Moon, S., Koo, G., and Moon, G. (2015). Dimming-Feedback Control Method for TRIAC Dimmable LED Drivers. IEEE Transactions on industrial Electronics, 62(2), 960-964.

Olabo, O., Ochayi, A., Blessing, O., Aderogba, A., and Ayoola, S. (2021). The Design, Construction, and Expert Appraisal of A 220v Inverter. ASEAN Journal of Science and Engineering, 1(3), 183-190.

Sien, C., Jen, Y., and Ching, W. (2012). An Efficient Driver for Dimmable LED Lighting. IEEE Transactions on Power Electronics, 27(11), 4613-4618.

Suroto, N. (2021). Economic Analysis: Solar Panels Application of NFT (Nutrient Film Technique) Hydroponic System in Bandung. ASEAN Journal of Science and Engineering Education, 1(1), 21-30.

Yu, Y., Zhang, K., Liu, H., Yang, L., and Zhang, D. (2020). Real-Time Visual Localization of the Picking Points for a Ridge-Planting Strawberry Harvesting Robot. IEEE Access, 8(2020), 116556 - 116567. 